\title{
The Rule for Evolution of Cooperation in Quantum Games
}

\author{
Z.W. SUN* \\ Institute of Applied Sciences, Shanghai Dianji University, Shanghai 200240, PR China
}

(Received March 4, 2009; in final form May 14, 2009)

\begin{abstract}
Cooperation is essential to biological organizations and human society. In this paper, the conditions of dilemmas resolving by quantum strategies in the three games, Prisoners' Dilemma, Chicken Game and Stag Hunt, are presented. The rule for the evolution of cooperation in general quantum games, $b / c>\operatorname{coth}^{2} \gamma$, is put forward. The formation of the rule is similar to those of the five rules in the classical game theory summarized by Nowak. $b / c$ is still the benefit-to-cost ratio of the altruistic act, while $\gamma$ is a measure for the game's entanglement. The rule is the prerequisite for the realization of cooperation in quantum games.
\end{abstract}

PACS numbers: 02.50.Le, 03.67.-a, 87.23.-n

\section{Introduction}

Recently, there are two focal topics in game theory. One is concerned with mechanisms of cooperation [1-3]. Cooperation is essential to the existence and stability of biological organizations and human society [4-7]. Individual cells rely on the cooperation among their components. Multicellular organisms exist as a result of the cooperation among their cells. Social insects, such as ants and bees, also live by cooperation. Human beings may be the champions of cooperation. From hunter-gatherer societies to nation-states, cooperation, in a sense, is the decisive organizing principle of human society. Cooperation is indispensable to the construction of a high level of organization as well. The very origin of life and the advent of human languages are all based on cooperation.

However, cooperation is always vulnerable to exploitation by defectors. In the absence of a specific mechanism for the evolution of cooperation, natural selection favors defectors. A key assumption in the classical game theory is that every player in games is perfectly rational when he selects his strategies to maximize his payoff $[8,9]$. The rationality leads players to selfish behavior. Every gene, every cell, and every organism does its best to promote its own evolutionary success at the expense of its competitors. Therefore the evolution of cooperation requires specific mechanisms. One central objective of the evolutionary game theory is to understand the workings of cooperative behaviors of individuals in an ecosystem [10-12]. There are at least five mechanisms so far [13-27] that can guarantee the evolution of cooperation when their requirements are fulfilled, as are shown in Table II.

Another focal topic is extending game theory into quantum domain. As more and more interest falls in

* e-mail: sunzw@sdju.edu.cn quantum computing and quantum information theory $[28,29]$, games are studied in quantum version so that dilemmas can be resolved. Many interesting results have been put forward, for the concepts of quantum mechanics, e.g., quantum superpositions, entanglement and quantum operations, are exploited [30-37]. Meyer studied the PQ penny flip game in quantum world and showed that a player implementing quantum strategies would always defeat his opponent playing classical strategies [30]. Eisert et al. quantized Prisoners' Dilemma (PD) and demonstrated that the dilemma could be escaped [31]. Marinatto and Weber investigated the Battle of the Sexes game in quantum domain and found a unique equilibrium if entangled quantum strategies were allowed [32]. Lee and Johnson reported that quantum games looked more efficient than classical ones [33]. Du et al. realized quantum PD on their nuclear magnetic resonance quantum computer [34]. Possible applications of quantum games in biology were thoroughly discussed by Iqbal et al. $[35,36]$, in economics by Piotrowski and Sladkowski [37]. It is obvious that the quantum game theory is paid more and more attention to though it has not been accepted by scientists at all [38-41]. One can refer to Iqbal's and Rosero's theses for a general review on the quantum game theory $[42,43]$.

The quantum game theory offers a new mechanism for the evolution of cooperation. It is natural to ask whether there is a rule in the quantum game theory, which is similar to the ones in the classical game theory. In Ref. [31], two players in a maximally entangled quantum game can escape from PD. However, it is difficult for a maximally entangled quantum game to be realized in practice, for no quantum system can be completely isolated from its environment. System-environment interactions should induce decoherence of quantum systems [44-46]. The purpose of this paper is to look for the rule for the realization of cooperation in a general quantum game. We shall 
analyze the payoffs in non-maximally entangled quantum games so as to obtain their modified matrices, from which we can get what we want.

The remainder of this paper is organized as follows. In Sect. 2, the three dilemmas discussed in this paper and the rules for the evolution of cooperation in the classical game theory are introduced. In Sect. 3, the quantization scheme employed in this research is reviewed. Then the payoffs in non-maximally entangled quantum games are calculated. In Sect. 4, the modified matrices of some quantum games are obtained, from which the conditions of the corresponding dilemmas resolving and the rule for the evolution of cooperation in the quantum game theory are acquired. Finally, the results in this paper are concluded and commented on.

\section{Dilemmas and rules for cooperation in classical game theory}

Given that the status of cooperative players are often equal in games, only the dilemmas in $2 \times 2$ symmetric games will be discussed in this paper. The games can be classified into 78 types, of which four ones are in dilemmas [47]. They are PD, Chicken Game (CG), Stag Hunt (SH) and Deadlock Game (DG) [48], as are shown in Table I.

TABLE I

Payoff matrices for some games.

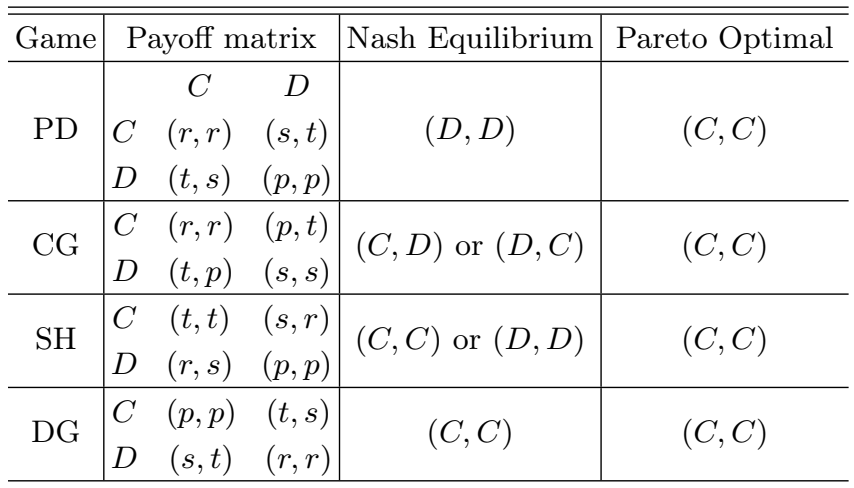

$t>r>p>s$. In matrices, strategy $C$ denotes cooperation, strategy $D$ defection. The first payoffs refer to the player adopting the row strategies, who is called the row player, while the second payoffs the player adopting the column strategies, who is called the column player.

PD has been the most influential in social sciences since the later half of the twentieth century. It is discussed most extensively to reveal the emergence of altruistic behaviors in communities consisting of selfish individuals. According to the classical game theory, either player in
PD should adopt strategy $D$ so that he can get more payoff no matter what his opponent's strategy is. Profile $(D, D)$ is the unique Nash equilibrium (NE) of the game, while profile $(C, C)$ is Pareto Optimal, which is the most efficient. Hence a dilemma occurs. In CG, the dilemma occurs because there are two NEs, $(C, D)$ and $(D, C)$. The players without communicating with each other cannot decide on which NE to choose. The dilemma in SH is very different from those in the above two games. $\mathrm{SH}$ has two NEs, $(C, C)$ and $(D, D)$. Obviously, strategy $C$ is better for both players. PD and CG are discussed on the assumption that players are rational, on which there exists no dilemma in SH. However, in this game, the dilemma can be found in the following sense: If one cooperates but the other player defects, then the former gets the worst payoff, while the latter still gets the second highest payoff. In other words, the dilemma arises from the fear that the other player might not be rational. We can transform PD into DG by exchanging strategies $C$ with $D$. DG can be studied in the way PD is. Therefore we merely consider the first three games.

If a mechanism can change the relations among the entries in the payoff matrices of the games, the dilemmas would be solved. Here we introduce several related concepts by taking $\mathrm{PD}$ as an example. (i) If $r>t, C$ is evolutionary stable strategy (ESS). An infinitely large population of cooperators cannot be invaded by defectors under deterministic selection dynamics [9]. (ii) If $r+s>t+p, C$ is risk-dominant (RD). If both strategies are ESS, then the risk-dominant strategy has the bigger basin of attraction. (iii) If $r+2 s>t+2 p, C$ is advantageous (AD). This concept is important to stochastic game dynamics in finite populations [49]. (iv) If $r>t$ and $s>p, C$ dominates $D$.

The above-mentioned five mechanisms which lead to the evolution of cooperation are kin selection [13-15], direct reciprocity [16-18], indirect reciprocity [19, 20], network reciprocity [21-23] and group selection [24-26]. All of them adopt payoff matrix (1), in which only the row player's payoffs are listed

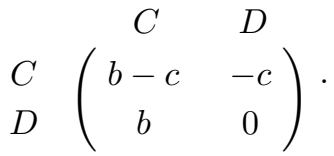

Here, $b>c>0$. Matrix (1), which is equal to a simplified PD with $r+s=t+p$, corresponds to cooperation in meaning that a donor pays a cost $c$ for a recipient to get a benefit $b$. Nowak [27] summarized up the rules of the five mechanisms [13-27], as are shown in Table II. Each rule is displayed as an inequality, in which the benefit-to-cost ratio of the altruistic act is greater than some critical value. 
TABLE II

Five rules for the evolution of cooperation.

\begin{tabular}{|c|c|c|c|c|c|c|}
\hline & \multicolumn{2}{|c|}{ Payoff matrix } & \multicolumn{3}{|c|}{ Cooperation is ... } \\
\hline & & $\mathrm{C}$ & $\mathrm{D}$ & ESS & $\mathrm{RD}$ & $\mathrm{AD}$ \\
\hline $\begin{array}{l}\text { Kin } \\
\text { selection }\end{array}$ & $\begin{array}{l}\mathrm{C} \\
\mathrm{D}\end{array}$ & $\begin{array}{c}(b-c)(1+r) \\
b-r c\end{array}$ & $\begin{array}{c}b r-c \\
0\end{array}$ & $\frac{b}{c}>\frac{1}{r}$ & $\frac{b}{c}>\frac{1}{r}$ & $\frac{b}{c}>\frac{1}{r}$ \\
\hline $\begin{array}{l}\text { Direct } \\
\text { reciprocity }\end{array}$ & \begin{tabular}{l|}
$\mathrm{C}$ \\
$\mathrm{D}$
\end{tabular} & $\begin{array}{c}(b-c) /(1-w) \\
b\end{array}$ & $\begin{array}{c}-c \\
0\end{array}$ & $\frac{b}{c}>\frac{1}{w}$ & $\frac{b}{c}>\frac{2-w}{w}$ & $\frac{b}{c}>\frac{3-2 w}{w}$ \\
\hline $\begin{array}{l}\text { Indirect } \\
\text { reciprocity }\end{array}$ & \begin{tabular}{l|}
$\mathrm{C}$ \\
$\mathrm{D}$
\end{tabular} & $\begin{array}{l}(b-c) \\
b(1-q)\end{array}$ & $\begin{array}{c}-c(1-q) \\
0\end{array}$ & $\frac{b}{c}>\frac{1}{q}$ & $\frac{b}{c}>\frac{2-q}{q}$ & $\frac{b}{c}>\frac{3-2 q}{q}$ \\
\hline $\begin{array}{l}\text { Network } \\
\text { reciprocity }\end{array}$ & $\begin{array}{l}\mathrm{C} \\
\mathrm{D}\end{array}$ & $\begin{array}{l}(b-c) \\
b-H\end{array}$ & $\begin{array}{c}H-c \\
0\end{array}$ & $\frac{b}{c}>k$ & $\frac{b}{c}>k$ & $\frac{b}{c}>k$ \\
\hline $\begin{array}{l}\text { Group } \\
\text { selection }\end{array}$ & \begin{tabular}{l|}
$\mathrm{C}$ \\
$\mathrm{D}$
\end{tabular} & $\begin{array}{c}(b-c)(m+n) \\
b n\end{array}$ & $\begin{array}{c}(b-c) m-c n \\
0\end{array}$ & $\frac{b}{c}>1+\frac{n}{m}$ & $\frac{b}{c}>1+\frac{n}{m}$ & $\frac{b}{c}>1+\frac{n}{m}$ \\
\hline
\end{tabular}

$r$ - genetic relatedness, $w$ - probability of next round,

$q$ - social acquaintanceship, $k$ - number of neighbors,

$n$ - group size, $m$ - number of groups.

\section{Quantization scheme and payoffs in quantum games}

\subsection{Eisert et al.'s quantization scheme}

Two schemes for the quantum game theory have been proposed [31, 32]. Our work is based on Eisert et al.'s scheme, by which PD is discussed, as is shown in Fig. 1. Each player has a qubit and can manipulate it independently. The quantum formulation proceeds by assigning the possible outcomes of the classical strategies $D$ and $C$ to two basis vectors,

$$
|C\rangle=\left(\begin{array}{l}
1 \\
0
\end{array}\right), \quad|D\rangle=\left(\begin{array}{l}
0 \\
1
\end{array}\right),
$$

which belong to the Hilbert space. At each instance, the state of the game is described by a vector in the tensor product space which is spanned by the classical game basis $|C C\rangle,|C D\rangle,|D C\rangle$ and $|D D\rangle$.

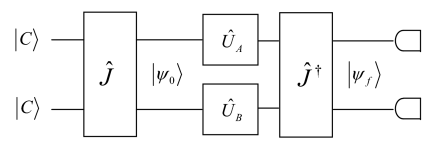

Fig. 1. The setup of a two player quantum game.

At the beginning of the game, the qubits $|C\rangle \otimes|C\rangle$ go through an entangling gate $\hat{J}=\exp (\mathrm{i} \gamma \hat{D} \otimes \hat{D} / 2)$, which is a reversible two-bit gate with $\gamma \in[0, \pi / 2]$. Therefore, $\left|\psi_{0}\right\rangle=\hat{J}|C C\rangle=\cos (\gamma / 2)|C C\rangle+\mathrm{i} \sin (\gamma / 2)|D D\rangle$. Since the entropy of $\left|\psi_{0}\right\rangle$ is $S=-\sin ^{2}(\gamma / 2) \ln \sin ^{2}(\gamma / 2)-$ $\cos ^{2}(\gamma / 2) \ln \cos ^{2}(\gamma / 2)$, the parameter $\gamma$ can be considered as a measure for the game's entanglement. $\hat{U}_{A}$ and $\hat{U}_{B}$ are the strategy moves available to the players, which belong to a subset $S$ of quantum unitary $2 \times 2$ matrices, i.e., the player's strategic space is restricted to a two-parameter set,

$$
\hat{U}(\theta, \phi)=\left(\begin{array}{cc}
\mathrm{e}^{\mathrm{i} \phi} \cos \theta / 2 & \sin \theta / 2 \\
-\sin \theta / 2 & \mathrm{e}^{-\mathrm{i} \phi} \cos \theta / 2
\end{array}\right),
$$

with $0 \leq \theta \leq \pi$ and $0 \leq \phi \leq \pi / 2$. Having executed their moves, which leave the game in a state $\left(\hat{U}_{A} \otimes \hat{U}_{B}\right) \hat{J}|C C\rangle$, two players forward their qubits for the final measurement, which is accomplished by a gate reversible $\tilde{J}$. $\tilde{J}=\hat{J}^{+}$. After the actions of both players and the gate $\tilde{J}$, the final state $\left|\psi_{f}\right\rangle=\tilde{J}\left(\hat{U}_{A} \otimes \hat{U}_{B}\right) \hat{J}|C C\rangle$ is a superposition. Measurement will make the final state collapse to one of classical outcomes and the payoff is returned according to the corresponding entries in the matrix. The row player's expected payoff is given by

$$
S_{A}=r P_{C C}+s P_{C D}+t P_{D C}+p P_{D D},
$$

where $P_{\sigma \sigma^{\prime}}=\left|\left\langle\sigma \sigma^{\prime} \mid \psi_{f}\right\rangle\right|^{2}$ is the probability that the final state will collapse into $\left|\sigma \sigma^{\prime}\right\rangle$. The results in two special instances are shown as follows:

(i) For a separable game with $\gamma=0$, there exists a pair of quantum strategies $(\hat{D}, \hat{D})$,

$$
\hat{D}=\hat{U}(\pi, 0)=\left(\begin{array}{cc}
0 & 1 \\
-1 & 0
\end{array}\right),
$$

which is a NE and yields payoff $(p, p)$. The quantum game behaves as a classical one.

(ii) For a maximally entangled quantum game with $\gamma=\pi / 2$, a novel $\mathrm{NE}(\hat{Q}, \hat{Q})$,

$$
\hat{Q}=\hat{U}(0, \pi / 2)=\left(\begin{array}{cc}
\mathrm{i} & 0 \\
0 & -\mathrm{i}
\end{array}\right),
$$

exists, which yields payoff $(r, r)$ and has the property of being Pareto Optimal. Therefore the dilemma existing in the classical game is removed. 


\subsection{Payoffs in non-maximally entangled quantum games}

If a quantum game is not maximally entangled, the quantization process of the game can be depicted in the matrix as follows:

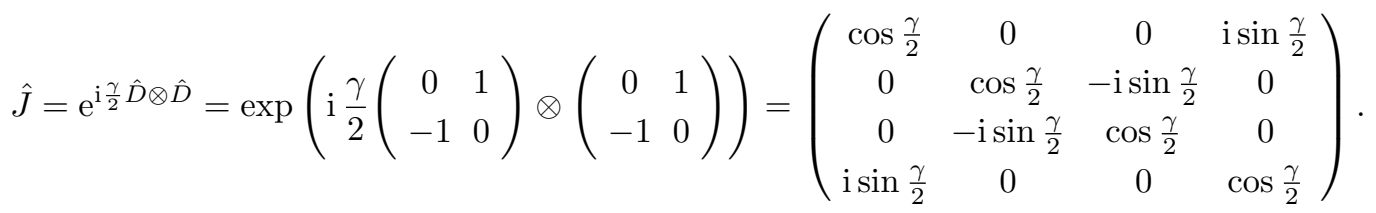

Therefore,

As

$$
\left|\psi_{0}\right\rangle=\hat{J}|\hat{C} \hat{C}\rangle=\left(\begin{array}{c}
\cos \frac{\gamma}{2} \\
0 \\
0 \\
\text { i } \sin \frac{\gamma}{2}
\end{array}\right)
$$

$$
\begin{aligned}
& \hat{U}_{A} \otimes \hat{U}_{B}=\left(\begin{array}{cc}
\mathrm{e}^{\mathrm{i} \phi_{A}} \cos \frac{\theta_{A}}{2} & \sin \frac{\theta_{A}}{2} \\
-\sin \frac{\theta_{A}}{2} & \mathrm{e}^{-\mathrm{i} \phi_{A}} \cos \frac{\theta_{A}}{2}
\end{array}\right) \otimes\left(\begin{array}{cc}
\mathrm{e}^{\mathrm{i} \phi_{B}} \cos \frac{\theta_{B}}{2} & \sin \frac{\theta_{B}}{2} \\
-\sin \frac{\theta_{B}}{2} & \mathrm{e}^{-\mathrm{i} \phi_{B}} \cos \frac{\theta_{B}}{2}
\end{array}\right), \\
& \hat{U}_{A} \otimes \hat{U}_{B} \hat{J}|\hat{C} \hat{C}\rangle=\left(\begin{array}{c}
\mathrm{e}^{\mathrm{i}\left(\phi_{A}+\phi_{B}\right)} \cos \frac{\theta_{A}}{2} \cos \frac{\theta_{B}}{2} \cos \frac{\gamma}{2}+\mathrm{i} \sin \frac{\theta_{A}}{2} \sin \frac{\theta_{B}}{2} \sin \frac{\gamma}{2} \\
-\mathrm{e}^{\mathrm{i} \phi_{A}} \cos \frac{\theta_{A}}{2} \sin \frac{\theta_{B}}{2} \cos \frac{\gamma}{2}+\mathrm{i} e^{-\mathrm{i} \phi_{B}} \sin \frac{\theta_{A}}{2} \cos \frac{\theta_{B}}{2} \sin \frac{\gamma}{2} \\
-\mathrm{e}^{\mathrm{i} \phi_{B}} \sin \frac{\theta_{A}}{2} \cos \frac{\theta_{B}}{2} \cos \frac{\gamma}{2}+\mathrm{i} e^{-\mathrm{i} \phi_{A}} \cos \frac{\theta_{A}}{2} \sin \frac{\theta_{B}}{2} \sin \frac{\gamma}{2} \\
\sin \frac{\theta_{A}}{2} \sin \frac{\theta_{B}}{2} \cos \frac{\gamma}{2}+\mathrm{ie}^{-\mathrm{i}\left(\phi_{A}+\phi_{B}\right)} \cos \frac{\theta_{A}}{2} \cos \frac{\theta_{B}}{2} \sin \frac{\gamma}{2}
\end{array}\right) \\
& \left|\psi_{f}\right\rangle=\hat{J}^{+} \hat{U}_{A} \otimes \hat{U}_{B} \hat{J}|\hat{C} \hat{C}\rangle=\left(\begin{array}{c}
\cos \left(\phi_{A}+\phi_{B}\right) \cos \frac{\theta_{A}}{2} \cos \frac{\theta_{B}}{2}+\mathrm{i} \sin \left(\phi_{A}+\phi_{B}\right) \cos \frac{\theta_{A}}{2} \cos \frac{\theta_{B}}{2} \cos \gamma \\
\sin \phi_{B} \sin \frac{\theta_{A}}{2} \cos \frac{\theta_{B}}{2} \sin \gamma-\cos \phi_{A} \cos \frac{\theta_{A}}{2} \sin \frac{\theta_{B}}{2}-\mathrm{i} \sin \phi_{A} \cos \frac{\theta_{A}}{2} \sin \frac{\theta_{B}}{2} \cos \gamma \\
\sin \phi_{A} \cos \frac{\theta_{A}}{2} \sin \frac{\theta_{B}}{2} \sin \gamma-\cos \phi_{B} \sin \frac{\theta_{A}}{2} \cos \frac{\theta_{B}}{2}-\mathrm{i} \sin \phi_{B} \sin \frac{\theta_{A}}{2} \cos \frac{\theta_{B}}{2} \cos \gamma \\
\sin \left(\phi_{B}+\phi_{B}\right) \cos \frac{\theta_{A}}{2} \cos \frac{\theta_{B}}{2} \sin \gamma+\sin \frac{\theta_{A}}{2} \sin \frac{\theta_{B}}{2}
\end{array}\right)
\end{aligned}
$$

The probabilities that the final state will collapse into the basis vectors of the tensor product space are

$$
\begin{aligned}
P_{C C} & =\cos ^{2}\left(\phi_{A}+\phi_{B}\right) \cos ^{2} \frac{\theta_{A}}{2} \cos ^{2} \frac{\theta_{B}}{2}+\sin ^{2}\left(\phi_{A}+\phi_{B}\right) \cos ^{2} \frac{\theta_{A}}{2} \cos ^{2} \frac{\theta_{B}}{2} \cos ^{2} \gamma, \\
P_{C D} & =\left(\sin \phi_{B} \sin \frac{\theta_{A}}{2} \cos \frac{\theta_{B}}{2} \sin \gamma-\cos \phi_{A} \cos \frac{\theta_{A}}{2} \sin \frac{\theta_{B}}{2}\right)^{2}+\sin ^{2} \phi_{A} \cos ^{2} \frac{\theta_{A}}{2} \sin ^{2} \frac{\theta_{B}}{2} \cos ^{2} \gamma, \\
P_{D C} & =\left(\sin \phi_{A} \cos \frac{\theta_{A}}{2} \sin \frac{\theta_{B}}{2} \sin \gamma-\cos \phi_{B} \sin \frac{\theta_{A}}{2} \cos \frac{\theta_{B}}{2}\right)^{2}+\sin ^{2} \phi_{B} \sin ^{2} \frac{\theta_{A}}{2} \cos ^{2} \frac{\theta_{B}}{2} \cos ^{2} \gamma, \\
P_{D D} & =\left(\sin \left(\phi_{B}+\phi_{B}\right) \cos \frac{\theta_{A}}{2} \cos \frac{\theta_{B}}{2} \sin \gamma+\sin \frac{\theta_{A}}{2} \sin \frac{\theta_{B}}{2}\right)^{2} .
\end{aligned}
$$

We can get the payoffs of the non-maximally entangled quantum game by Eqs. (4) and (12).

\section{Rule for evolution of cooperation in quantum games}

\subsection{Conditions of dilemmas resolving in quantum games}

It is known by Eqs. (4) and (12) that the payoff of profile $(\hat{Q}, \hat{Q})$ is equal to that of profile $(\hat{C}, \hat{C})$. Here, we adopt $\hat{Q}$ as cooperator's strategy instead of $\hat{C}$ so that the dilemmas in the classical game theory can be resolved in the quantum game theory.

For PD, the modified matrix in the non-maximally entangled quantum game can be obtained

$$
\left.\hat{Q} \quad \begin{array}{cc}
\hat{Q} & \hat{D} \\
r & t \sin ^{2} \gamma+s \cos ^{2} \gamma \\
t \cos ^{2} \gamma+s \sin ^{2} \gamma & p
\end{array}\right) .
$$

PD is resolved if $r>t \cos ^{2} \gamma+s \sin ^{2} \gamma$ and $p<t \sin ^{2} \gamma+$ $s \cos ^{2} \gamma$. In these conditions, a unique NE, $(\hat{Q}, \hat{Q})$, exists, which is Pareto Optimal. The two inequalities call for

$$
\sin ^{2} \gamma>\max \left(\sqrt{\frac{t-r}{t-s}}, \sqrt{\frac{p-s}{t-s}}\right),
$$

which is considered as the condition of $\mathrm{PD}$ resolving. When $t, r, p$ and $s$ are equal to $5,3,1$ and 0 respectively, $\sin ^{2} \gamma$ is greater than $\sqrt{2 / 5}$. 
In the same way, we can get the modified matrix of CG,

$$
\left.\hat{Q} \quad \begin{array}{cc}
\hat{Q} & \hat{D} \\
r & t \sin ^{2} \gamma+p \cos ^{2} \gamma \\
t \cos ^{2} \gamma+p \sin ^{2} \gamma & s
\end{array}\right),
$$

and the condition of escaping from the dilemma of CG,

$$
\sin ^{2} \gamma>\sqrt{\frac{t-r}{t-p}}
$$

as well as the modified matrix of $\mathrm{SH}$,

$$
\left.\hat{Q} \quad \begin{array}{cc}
\hat{Q} & \hat{D} \\
t & r \sin ^{2} \gamma+s \cos ^{2} \gamma \\
r \cos ^{2} \gamma+s \sin ^{2} \gamma & p
\end{array}\right),
$$

and the condition of escaping from the dilemma of $\mathrm{SH}$,

$$
\sin ^{2} \gamma>\sqrt{\frac{p-s}{r-s}} .
$$

The NEs of the three quantum games are $(\hat{Q}, \hat{Q})$ when their conditions are fulfilled.

\subsection{Rule for the evolution of cooperation in quantum games}

In order to get a rule for the evolution of cooperation in quantum games, which can be compared with Nowak's, the modified matrix of matrix (1) in the non-maximally entangled quantum game is provided,

$$
\begin{aligned}
& \hat{Q} \\
& \begin{array}{l}
\hat{Q} \\
\hat{D}
\end{array}\left(\begin{array}{cc}
b-c & b \sin ^{2} \gamma-c \cos ^{2} \gamma \\
b \cos ^{2} \gamma-c \sin ^{2} \gamma & 0
\end{array}\right) .
\end{aligned}
$$

For this payoff matrix, $\hat{Q}$ is ESS, RD and $\mathrm{AD}$ if $b / c>$ $\operatorname{coth}^{2} \gamma$. In this condition, $\hat{Q}$ dominates $\hat{D}$, and profile $(\hat{Q}, \hat{Q})$ is Pareto Optimal. The cooperation between two players is realized. Therefore the condition, $b / c>\operatorname{coth}^{2} \gamma$, is considered as the rule for evolution of cooperation in quantum games.

\section{Conclusion}

The quantum game theory provides an effective way to realize cooperation. It will be valued more and more with the development of quantum information theory. In this paper, not only the conditions of three dilemmas resolving in non-maximally entangled quantum games are acquired, but also the rule for the evolution of cooperation in the quantum games is gotten, which corresponds to the rules in the classical game theory. The rule, $b / c>\operatorname{coth}^{2} \gamma$, ensures that strategy $\hat{Q}$ dominates strategy $\hat{D}$ so that cooperation can also be realized in non-maximally entangled quantum games. The rule, which can no more be replaced by each of the five rules in Ref. [27] than the mechanics of quantum game can by the one of the classical game, makes the mechanics of cooperation more substantial. Although one may claim that the quantum PD is not a genuine quantum game [38], the rule obtained in this paper is expected to offer a way of resolving dilemmas in decision science or economics and to serve as a tool of investigating the cooperation behaviors in the games on the molecular level, such as replication of DNA and synthesis of proteins.

As a referee pointed out, it is very interesting to extend the rule for the evolution of cooperation to multi-player games, for cooperation is more and more important in games involving many players. This issue needs some more preparations in mathematics, which cannot be realized in this paper. It will be addressed in our future work.

\section{Acknowledgments}

We would like to use this opportunity to thank the anonymous referees for their valuable comments and instructive suggestions, which greatly improved the paper. This work was supported by Shanghai Education Committee under grant 06 A109 and by Shanghai Dianji University under grant $08 \mathrm{C} 405$.

\section{References}

[1] P.D. Taylor, T. Day, G. Wild, Nature 447, 05784 (2007).

[2] M.A. Nowak, A. Sasaki, C. Taylor, D. Fudenberg, Nature 428, 646 (2004).

[3] A. Traulsen, M.A. Nowak, Proc. Natl. Acad. Sci. USA 103, 10952 (2006).

[4] L.A. Dugatkin, Cooperation Among Animals, Oxford University Press, Oxford 1997.

[5] E. Fehr, U. Fischbacher, Nature 425, 785 (2003).

[6] M.A. Nowak, K. Sigmund, Nature 437, 1291 (2005).

[7] K.R. Foster, Nature 441, 291 (2006).

[8] J.M. Smith, Evolution and the Theory of Games, Cambridge University Press, Cambridge 1982.

[9] J. Hofbauer, K. Sigmund, Evolutionary Games and Population Dynamics, Cambridge University Press, Cambridge 1998.

[10] M. Doebeli, C. Hauert, T. Killingback, Science 306 , 859 (2004).

[11] M.A. Nowak, K. Sigmund, Science 303, 793 (2004).

[12] C. Hauert, A. Traulsen, H. Brandt, M.A. Nowak, K. Sigmund, Science 316, 1905 (2007).

[13] A.S. Griffin, S.A. West, Science 302, 634 (2003).

[14] K.R. Foster, T. Wenseleers, F. Ratnieks, Trends Ecol. Evol. 21, 57 (2006).

[15] P.D. Taylor, G. Wild, A. Gardner, J. Evol. Biol. 20, 296 (2007).

[16] R. Axelrod, W.D. Hamilton, Science 211, 1390 (1981).

[17] M.A. Nowak, K. Sigmund, Nature 364, 56 (1993).

[18] L.A. Imhof, D. Fudenberg, M.A. Nowak, Proc. Natl. Acad. Sci. USA 102, 10797 (2005).

[19] M.A. Nowak, K. Sigmund, Nature 393, 573 (1998).

[20] H. Brandt, K. Sigmund, Proc. Natl. Acad. Sci. USA 102, 2666 (2005).

[21] M.A. Nowak, R.M. May, Nature 359, 826 (1992). 
[22] G. Szabo, C. Hauert, Phys. Rev. Lett. 89, 118101 (2002).

[23] H. Ohtsuki, C. Hauert, E. Lieberman, M.A. Nowak, Nature 441, 502 (2006).

[24] J.A. Fletcher, M. Zwick, J. Theor. Biol. 228, 303 (2004).

[25] A. Traulsen, A.M. Sengupta, M.A. Nowak, J. Theor. Biol. 235, 393 (2005).

[26] S. Bowles, Science 314, 1569 (2006).

[27] M.A. Nowak, Science 314, 1560 (2006).

[28] M.A. Nielsen, I.L. Chuang, Quantum Computation and Quantum Information, Cambridge University Press, Cambridge 2000.

[29] C.P. Williams, S.H. Clearwater, Explorations in Quantum Computing, Springer, New York 1998.

[30] D.A. Meyer, Phys. Rev. Lett. 82, 1052 (1999).

[31] J. Eisert, M. Wilkins, M. Lewenstein, Phys. Rev. Lett. 83, 3077 (1999).

[32] T. Marinatto, A. Weber, Phys. Lett. A 272, 291 (2000).

[33] C.F. Lee, N.F. Johnson, Phys. Rev. A 67, 022311 (2003).

[34] J. Du, H. Li, X. Xu, M. Shi, J. Wu, X. Zhou, R. Han, Phys. Rev. Lett. 88, 137902 (2002).

[35] A. Iqbal, A.H. Toor, Commun. Theor. Phys. 3, 335 (2004).
[36] A. Iqbal, T. Cheon, D. Abbott, Phys. Lett. A $\mathbf{3 7 2}$, 6564 (2008).

[37] E.W. Piotrowski, J. Sladkowski, Physica A 318, 516 (2003).

[38] S.J. van Enk, Phys. Rev. Lett. 84, 789 (2000).

[39] S.J. van Enk, R. Pike, Phys. Rev. A 66, 024306 (2002).

[40] S.C. Benjamin, P.M. Hayden, Phys. Rev. Lett. 87, 069801 (2001).

[41] S.C. Benjamin, Phys. Lett. A 277, 180 (2000).

[42] A. Iqbal, quant-ph/0503176, preprint, 2006.

[43] A.F.H. Rosero, quant-ph/0402117, preprint, 2004.

[44] E. Joos, H.D. Zeh, C. Kiefer, Decoherence and the Appearance of a Classical World in Quantum Theory, 2nd ed., Springer, Berlin 2003.

[45] K.L. Chen, H. Ang, D. Kiang, L.C. Kwek, C.F. Lo, Phys. Lett. A 316, 317 (2003).

[46] A. Nawaz, A.H. Toor, J. Phys. A, Math. Gen. 39, 9321 (2006).

[47] A. Rapoport, M.J. Guyer, D.G. Gordon, The $2 \times 2$ Game, Univ. of Michigan press, Michigan 1976.

[48] R.B. Myerson, Game Theory: An Analysis of Conflict, MIT Press, Cambridge 1991.

[49] H. Ohtsukia, P. Bordalob, M.A. Nowak, J. Theor. Biol. 249, 289 (2007). 